\title{
GFAP expression is regulated by Pax3 in brain glioma stem cells
}

\author{
XING SU $^{1 *}$, XIAOJIANG LIU ${ }^{1 *}$, LANCHUN NI $^{1}$, WEI SHI ${ }^{1}$, HUI ZHU ${ }^{1}$, JINLONG SHI $^{1}$, JIAN CHEN $^{1}$, \\ ZHIKAI GU ${ }^{1}$, YILU GAO ${ }^{1}$, QING LAN ${ }^{2}$ and QINGFENG HUANG ${ }^{1}$ \\ ${ }^{1}$ Department of Neurosurgery, Affiliated Hospital of Nantong University, Nantong, Jiangsu 226001;
${ }^{2}$ Department of Neurosurgery, Second Affiliated Hospital of Soochow University, Suzhou, Jiangsu 215000, P.R. China
}

Received December 4, 2015; Accepted January 6, 2016

DOI: $10.3892 /$ or.2016.4917

\begin{abstract}
Glioblastomas are understood to evolve from brain glioma stem cells (BGSCs), and yet the biology underlying this model of tumorigenesis is largely unknown. Paired box 3 protein $(\mathrm{Pax} 3)$ is a member of the paired box (Pax) family of transcription factors that is normally expressed during embryonic development, but has recently been implicated in tumorigenesis. The present study demonstrated that $\operatorname{Pax} 3$ is differentially expressed in U87MG human glioma cell, BGSC and normal 1800 human astrocyte lines. Herein, we identified that the glial fibrillary acidic protein (GFAP), a major intermediate filament protein of mature astrocytes, is directly downregulated during the differentiation of BGSCs via the binding of Pax 3 to the promoter region of GFAP. Moreover, siRNA silencing of Pax 3 arrested BGSC differentiation, while overexpression of $\operatorname{Pax} 3$ promoted the differentiation in BGSCs. Furthermore, we studied the cell proliferation, invasion, apoptosis, differentiation and expression of Pax3 and GFAP in Pax3 siRNA-knockdown and Pax3-overexpressing BGSC models by CCK-8, Transwell migration, flow cytometry and western blot assays. The results indicate that Pax3 regulates GFAP expression, and that Pax 3 may contribute to the evolution of BGSCs towards malignancy.
\end{abstract}

\section{Introduction}

Malignant gliomas are the most common type of primary brain tumors (1), of which patients have a notably high likelihood of relapse after treatment (2-4). Glioblastomas are understood to

Correspondence to: Dr Qing Lan, Department of Neurosurgery, Second Affiliated Hospital of Soochow University, Suzhou, Jiangsu 215000, P.R. China

E-mail: szlq006@yahoo.com.cn

Dr Qingfeng Huang, Department of Neurosurgery, Affiliated Hospital of Nantong University, Nantong, Jiangsu 226001, P.R. China E-mail: hqf025@ntu.edu.cn

${ }^{*}$ Contributed equally

Key words: GFAP, Pax3, glioma, proliferation, differentiation, apoptosis evolve from brain glioma stem cells (BGSCs). BGSCs have the capabilities of self-renewal, and may render themselves profitable to the survival of tumors by conferring resistance to therapeutic treatments $(5,6)$. It was initially proposed that acute myelogenous leukemia may evolve from a subset of precursor stem cells, and considerable efforts have been devoted to studying this sub-population of cells in numerous cancers since (7). Stem cells, for example, exhibit many of the hallmarks inherent to malignant tumors, including the abilities to proliferate, invade and metastasize (5).

Stem cells expressing the CD133 membrane protein are markedly pronounced within cells of malignant gliomas $(7,8)$. Furthermore, the encephalic inoculation of $\mathrm{CD}_{133^{+}}$stem cells into NOD-SCID mice results in tumor growth (9). Thus, $\mathrm{CD}_{133^{+}}$stem cells are likely to be important for tumor recurrence. Paired box 3 protein $(\operatorname{Pax} 3)$ is a member of the paired box (Pax) family of transcription factors that is normally expressed during embryonic development, but has recently been implicated in tumorigenesis (8). The expression of Pax3, for example, was found to be positively correlated with melanoma progression (10-12). In addition, GFAP is a major intermediate filament protein, where it likely plays a critical role in mature astrocytes owing to its high abundance and strong conservation among vertebrates. Pax 3 has previously been reported to negatively regulate the expression of GFAP during the differentiation of astrocytes from neural stem cells (NSCs) $(13,14)$. In addition, Pax 3 overexpression can be detected during the process of the astrocyte precursor cell proliferation and can maintain the high malignancy in gliomas by negatively regulating GFAP expression in glioma cells (15). Thus, we speculated that GFAP is a Pax3-responsive gene in BGSCs. In these studies, we attempted to examine the expression of Pax3 and to determine whether Pax 3 could bind to the element of the GFAP promoter in BGSCs.

\section{Materials and methods}

Cell lines and reagents. Human BGSCs were kindly gifted by Soochow University, China. Human malignant glioma cell line (U87MG) and normal human astrocytes (1800) were obtained from the Cell Library of the Chinese Academy of Sciences (Shanghai, China). BGSCs were cultured at $37^{\circ} \mathrm{C}$ in $5 \% \mathrm{CO}_{2}$ in a 1:1 mix of Dulbecco's modified Eagle's medium and Ham's F-12 medium (DMEM/F-12) (Gibco, Grand Island, NY, USA) containing $10 \mathrm{ml} \mathrm{B} 27,10 \mu \mathrm{g}$ EGF, $10 \mu \mathrm{g}$ FGF, 
$5 \mathrm{ml} \mathrm{L}$-glutamine, $5 \mathrm{ml}$ MEM-vitamin solution, $5 \mathrm{ml}$ sodium pyruvate, $5 \mathrm{ml}$ MEM non-essential amino acids (Gibco), and $5 \mathrm{ml}$ penicillin-streptomycin solution (HyClone, Logan City, UT, USA). U87MG cells were cultured at $37^{\circ} \mathrm{C}$ in $5 \% \mathrm{CO}_{2}$ in DMEM supplemented with $10 \%$ fetal bovine serum (FBS), $2 \mathrm{mM}$ L-glutamine and $100 \mathrm{U} / \mathrm{ml}$ penicillin-streptomycin (Gibco). The normal astroctyes (1800) were cultured at $37^{\circ} \mathrm{C}$ and $5 \% \mathrm{CO}_{2}$ in modified RPMI-1640 medium (HyClone) supplemented with $10 \%$ FBS, $2 \mathrm{mM}$ L-glutamine and $100 \mathrm{U} / \mathrm{ml}$ penicillin-streptomycin (Gibco). Culture medium was altered every 3-4 days. U87MG and 1800 cells were split using $0.25 \%$ trypsin. BGSCs were split using StemPro Accutase. The present study obtained ethical approval from the Affiliated Hospital of Nantong University, China [approval ID: (2013)040].

$R T-P C R$. Total RNA was extracted from cells with TRIzol (Invitrogen, Carlsbad, CA, USA), from which cDNA was reverse transcribed using the Omniscript RT kit (Qiagen) aaccording to the manufacturer's instructions. The sequences for primers used were as follows: H-Pax3-F, 5'-AAGCCCA AGCAGGTGACAA-3' and H-Pax3-R, 5'-ATGGAACTCAC TGACGGCAC-3'; H-GFAP-F, 5'-AGATCCGCACGCAGTA TGA-3' and H-GFAP-R, 5'-AGTCGTTGGCTTCGTGCTT-3'; H- $\beta$-actin-F, 5'-CATGAAGTGTGACGTGGACATC-3' and H- $\beta$-actin-R, 5'-GGACTCGTCATACTCCTGATTG-3'. The thermal cycles were: $95^{\circ} \mathrm{C}$ for $30 \mathrm{sec}, 68^{\circ} \mathrm{C}$ for $30 \mathrm{sec}$, and $72^{\circ} \mathrm{C}$ for $30 \mathrm{sec}$ for $35 \mathrm{cycles}$ for $\beta$-actin, and $95^{\circ} \mathrm{C}$ for $30 \mathrm{sec}, 58^{\circ} \mathrm{C}$ for $30 \mathrm{sec}$, and $72^{\circ} \mathrm{C}$ for $30 \mathrm{sec}$ for 35 cycles for Pax 3 .

Western blotting. Expression levels of $\beta$-actin, Pax 3 and GFAP proteins were determined via western blotting with specific antibodies as previously described (16). The following antibodies were used: primary antibodies against $\beta$-actin $(1: 2,000$ dilution), Pax3 (1:500) and GFAP (1:1,000); all of which were obtained from Abcam (Cambridge, MA, USA).

Immunofluorescence microscopy. Dispersed BGSCs were smeared onto a Poly-L-lysine glass slide. Cells were washed with phosphate-buffered saline (PBS), fixed in 4\% paraformaldehyde for $30 \mathrm{~min}$, permeabilized in $0.1 \%$ Triton X-100 for $30 \mathrm{~min}$, and blocked against non-specific binding in 5\% BSA (Amresco, Solon, OH, USA) for $45 \mathrm{~min}$ at room temperature. Subsequently, cells were incubated with rabbit anti-Pax3 polyclonal (1:100), GFAP monoclonal (1:100) (both from Abcam), anti-human nestin (1:100; Alexa Fluor 4881 eBioscience, San Diego, CA, USA), and anti-human CD133-APC antibodies (1:100; Miltenyi Biotec) overnight at $4^{\circ} \mathrm{C}$. The slides were then washes 3 times with PBS, and incubated with $\mathrm{Cy} 3$ or FITC fluorescently-labeled secondary antibodies for $2 \mathrm{~h}$ at room temperature. DNA was stained by incubating the slides in 4',6-diamidino-2-phenylindole (DAPI) $(0.2 \mathrm{mg} / \mathrm{ml})$ for $2 \mathrm{~min}$ immediately following incubation with secondary antibodies. Slides were stored at $4^{\circ} \mathrm{C}$ in the dark and visualized with a Leica fluorescence microscope (Germany). All assays were performed 3 times in duplicate.

siRNA transfection. Pax3 was knocked down as previously described (15), using siRNA sequences purchased from Biomics (Jiangsu, China). The siRNA sequences were: Pax3 sense,
5'-CGCAUCCUGAGAAGUAAAUdTdT-3' and Pax3 antisense, 5'-AUUUACUUCUCAGGAUGCGdTdT-3'; negative control (NC) sense, 5'-UUCUCCGAACGUGUCACG UTT-3' and NC antisense, 5'-ACGUGACACGUUCGGAGAA TT-3'. siRNAs were transfected into BGSCs in 6-well plates $(1 \mathrm{mg} / \mathrm{ml})$ using the MicroPoly-Transfecter Cell Reagent (Invitrogen) completed according to the manufacturer's instructions.

Transient transfections and differentiation. Plasmid vectors for overexpressing Pax3 were constructed by GeneChem (Shanghai, China). Transient transfections were carried out using the MicroPoly-Transfecter Cell Reagent according to the manufacturer's instructions. To induce differentiation, the transfected BGSCs were seeded onto coverslips coated with poly-L-lysine within 24-well plates, and cultured in DMEM/F-12 supplemented with $1 \%$ FBS for 3 days. Next, the neurospheres were fixed and processed for immunofluorescence microscopy as previously described. A subset of cells were omitted from immunofluorescence microscopy, and were incubated at $37^{\circ} \mathrm{C}$ for $24 \mathrm{~h}$ after transfection.

CCK-8 cell proliferation assay. Cells at $12 \mathrm{~h}$ post-transfection were seeded into 96 -well plates at a density of 4,000 cells/ well. Next, cell viability was determined using the Cell Counting Kit-8 (CCK-8; Dojindo, Shanghai, China). Briefly, $100 \mu \mathrm{l}$ DMEM/F-12 and $10 \mu \mathrm{l}$ CCK-8 reagent were added to each pre-cultured film, whereupon the plates were incubated for $1.5 \mathrm{~h}$ at $37^{\circ} \mathrm{C}$. The absorbance was determined at a wavelength of $450 \mathrm{~nm}$ using a Multiskan MK33 microplate reader (Thermo Electron Corporation, Shanghai, China).

Cell invasion assay. A cell invasion assay was performed using 24-well tissue culture plates (BD Biosciences, Bedford, MA, USA) consisting of an 8- $\mu \mathrm{m}$ polyethylene terephthalate membrane coated with a Matrigel basement membrane matrix $\left(100 \mu \mathrm{g} / \mathrm{cm}^{2}\right)$. In brief, the Matrigel (R\&D Systems, Minneapolis, MN, USA) was rehydrated overnight at $4^{\circ} \mathrm{C}$. The Transwell membranes were pre-coated with $24 \mathrm{mg} / \mathrm{ml}$ Matrigel. Two days after transfection, the cells $\left(5 \times 10^{4}\right)$ were seeded into the upper chamber with DMEM/F-12, and the lower chamber was filled with DMEM containing $10 \mathrm{ml} \mathrm{B27}$, $10 \mu \mathrm{g}$ EGF, $10 \mu \mathrm{g}$ FGF, $5 \mathrm{ml}$ L-glutamine, $5 \mathrm{ml}$ MEM-vitaminsolution, $5 \mathrm{ml}$ sodium pyruvate, $5 \mathrm{ml}$ MEM non-essential amino acids, and $5 \mathrm{ml}$ penicillin-streptomycin solution as a chemoattractant. The invasion assay was carried out in a $5 \% \mathrm{CO}_{2}$ humidified chamber at $37^{\circ} \mathrm{C}$ for $48 \mathrm{~h}$, and cells on the upper surface of the filters were removed by wiping the upper surface of the membrane with a cotton swab. The filter membrane was fixed in $4 \%$ paraformaldehyde, and stained with Coomassie blue. The degree of invasion was quantified by counting the cells that migrated to the lower side of the filter in at least six random fields at a magnification of $x 100$ using a fluorescence microscope. Experiments were repeated 3 times in triplicate.

Apoptosis detection. Cells were trypsinized, counted, washed twice with ice-cold PBS, and resuspended in $1 \mathrm{X}$ binding buffer (pH 7.4) containing $10 \mathrm{mM}$ HEPES/ $\mathrm{NaOH}, 140 \mathrm{mM} \mathrm{NaCl}$, and $2.5 \mathrm{mM} \mathrm{CaCl}_{2}$. Next, $100 \mu \mathrm{l}$ cell suspension was stained with $5 \mu$ l Annexin V-FITC fluorescent label (R\&D Systems 
Europe Ltd., Abingdon, UK) and $10 \mu 1$ propidium iodide (PI) (Sigma) for $15 \mathrm{~min}$ at room temperature in the dark. Finally, $400 \mu \mathrm{l}$ of binding buffer was added to each sample, which was immediately held on ice prior until analysis on a FACSCalibur flow cytometer (BD Pharmingen, San Diego, CA, USA), for which $10^{5}$ cells/sample were analyzed.

Monoclonal formation. The original neural cells were digested with StemPro Accutase into single cell suspension. Next, the digests were diluted to 40 cells $/ \mathrm{ml}$ with serum-free medium containing B27, bFGF and EGF, of which $50 \mu 1$ of this cell suspension was inoculated/microwell of a 96-well plate. Cells were centrifuged at $1,000 \mathrm{x}$ g, to which 3 parts supernatant was combined with 1 part fresh serum-free culture medium, and $\mathrm{bFGF}$ and EGF were added for a final concentration of $20 \mathrm{ng} / \mathrm{ml}$. Next, $100 \mu \mathrm{l}$ serum-free culture mixture was added to each microhole, whereupon cells were cultivated at $37^{\circ} \mathrm{C}$ and $5 \% \mathrm{CO}_{2}$. Finally, the division and cloning of the single cells were observed with microscopy.

Chromatin immunoprecipitation. Chromatin was immuneprecipitated with a ChIP assay kit (Upstate Biotechnology, Lake Placid, NY, USA) completed as per the manufacturer's instructions. Briefly, BGSCs were lysed, where upon the chromatin was immunoprecipitated with anti-Pax3 polyclonal antibodies (sc-376215; Santa Cruz Biotechnology, Inc., Santa Cruz, CA, USA).

Luciferase reporter assay. The length promoter plasmid pGFAP1600 of the human GFAP gene was cloned into a pGL3-Basic luciferase reporter vector (Promega, Madison, WI, USA) and amplified via PCR. The oligonucleotide PCR primer sequences used to detect the fragment of the pGFAP were as follows: GFAPF(KpnI), 5'-CCCGGTACCAAGCAGAC CTGGCAGCATTG-3' and GFAP R (HindIII), 5'-CGGAAGC TTGCCCGGGTGCCCCTGGCAAC-3'. Mutant-promoter constructs were created by deleting the nucleotides corresponding to the $\mathrm{P} 1$ and $\mathrm{P} 2$ probes (P1 F5, 5'-CATGCCCATGA CTCACCTTGGCACAG-3' and R5, 5'-GTGAGTCATGGGC ATGAAGAGGAGGC; P2 F7, 5'-GGGATTACGCCACCCCA CTCAGCCCT-3' and R7, 5'-GGGGTGGCGTAATCCCAGC ACTTTGG-3') of pGFAP1600, either individually or in combination, using a Pfu Turbo DNA polymerase from a QuikChange kit (Stratagene, La Jolla, CA, USA). The mutantpromoter constructs were termed pGFAP1600-DelP1 (deletion of 5 nucleotides within P1) or pGFAP1600-DelP2x (deletion of 8 nucleotides within P2). The human Pax3 was achieved by cloning the cDNA fragment into a pCI vector in HEK 293T cells. All constructs were confirmed by sequence validation. Normal or mutated GFAP promoter reporter plasmids were transfected together with the PRL (Promega) vector into HEK 293T cells using Lipofectamine 2000. HEK293T cells were used for luciferase assay since these cells are more easily transfected in comparison with BGSCs. Twenty-four hours after transfection, the cells were lysed and assayed using the Dual-Luciferase Assay kit (Promega). Promoter activity was measured by comparing luciferase levels.

Electrophoretic mobility shift assay. Putative interactions between Pax3 and GFAP mRNA transcripts were monitored via the electrophoretic mobility shift assay. Nuclear components of HEK 293T cells transfected with overexpression-Pax3 were extracted according to NE-PER Nuclear and Cytoplasmic Extraction Reagents (Pierce, Rockford, IL, USA) and electrophoretic mobility shift assays (EMSA) were exactly performed as previously described (17). First, we designed fluorescently-labeled normal and mutated probes. The sequence of the normal and mutated oligonucleotides used in this experiment were: $\mathrm{P} 1$ probe $\mathrm{Fw}$, 5'-ATGCCCAGTGAATGACTCAC-3' and Rw, 5'-GTGAGTCATTCACTGGGCAT-3'; PIM probe Fm, 5'-ATGCCCACTGTATGACTCAC-3' and Rm, 5'-GTGAGTCATACAGTGGGCAT-3'. For supershift experiments, nuclear extracts were pre-incubated with anti-Pax3 polyclonal antibodies (Abcam) before adding the labeled probes.

Statistical analysis. All statistical analyses, including the t-test was carried out using GraphPad Prism software (version 6; GraphPad Software, La Jolla, CA, USA).

\section{Results}

Identification of BGSCs and Pax3 and GFAP mRNA expression. We performed immunofluorescence staining with $\mathrm{CD}_{133}{ }^{+}$and nestin antibodies to identify BGSCs. Through immunofluorescence, the nuclei of the supposed BGSCs were stained blue, the cytoplasm red, and the cell membrane stained green, therefore confirming the expression of $\mathrm{CD}_{13}{ }^{+}$and nestin BGSC markers (Fig. 1A). We also determined whether Pax3 enhanced GFAP expression in the BGSCs using immunohistochemical staining (Fig. 1B), and observed that GFAP and Pax 3 proteins co-localized in the cytoplasm of the BGSCs. Furthermore, we measured the expression of Pax3 and GFAP in the U87MG human glioma cell, BGSC and 1800 normal human astrocyte lines by RT-PCR. Pax 3 was highly expressed in the BGSCs compared with that noted in the normal human astrocytes (Fig. 1C). In addition, we found that a single BGSC could form a colony of 50 BGSCs by proliferation, suggesting the observed colony formation resulted from the ability of BGSCs to self-renew and proliferate, rather than the recruitment of individual BGSCs (Fig. 1D).

Pax3 decreases the apoptosis, enhances the invasion and promotes the proliferation of BGSCs. The proportion of BGSCs undergoing apoptosis was determined using an Annexin V/PI apoptosis detection kit coupled to flow cytometry. We found that a significantly greater proportion of siPax3-transfected BGSCs underwent apoptosis relative to the control BGSCs $(\mathrm{P}<0.01)$, while the apoptotic index of BGSCs transfected to overexpress Pax3 was significantly lower than that of the untransfected cells $(\mathrm{P}<0.01$; Fig. $2 \mathrm{~A})$. Subsequently, we performed a cell invasion assay to determine the influence of Pax3 on the invasion of BGSCs. The number of invading BGSCs transfected with a Pax3-knockdown siRNA was significantly lower $(14 \pm 5)$ than that of the untransfected cells $(78 \pm 12 ; \mathrm{P}<0.01)$. Moreover, the number of BGSCs transfected with a Pax3-overexpression vector (152 \pm 6$)$ was markedly greater than that of the normal control cells $(\mathrm{P}<0.01)$. The above results suggest that Pax 3 enhances BGSC invasion in vivo (Fig. 2B). 


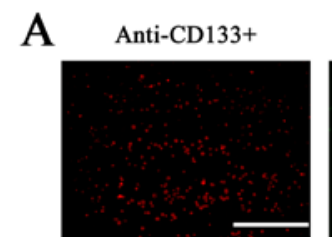

Anti-Nestin

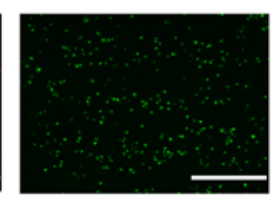

DAPI
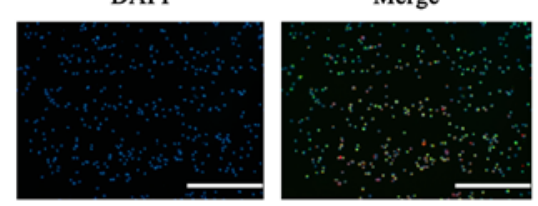

B
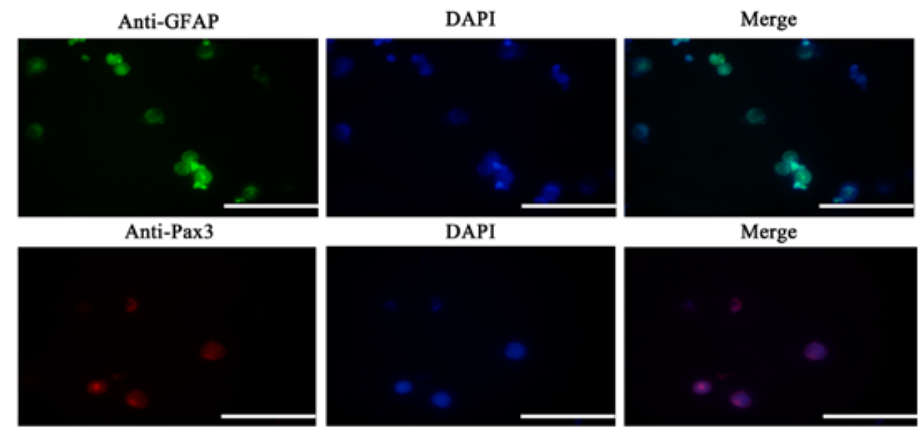

C
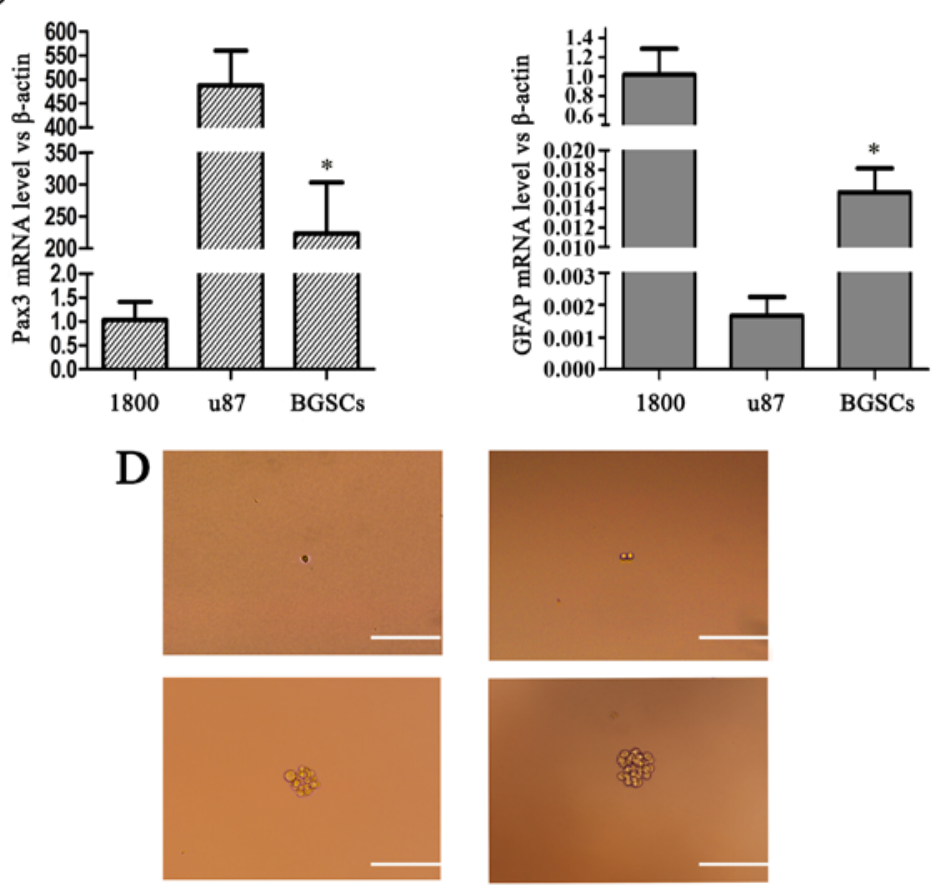

Figure 1. Correlation between GFAP and Pax3 expression in the BGSCs. (A) To confirm the presence of BGSCs, the cells were stained with anti-CD133+ and anti-nestin by immunofluorescence. (B) Immunofluorescence of Pax3 and GFAP protein was promoted in the BGSCs. Pax3 and GFAP proteins were colocalized in the BGSCs. (C) Expression levels of Pax 3 and GFAP mRNA were measured by quantitative RT-PCR and normalized to $\beta$-actin in the U87 cells, BGSCs and the astroglial 1800 cells. (D) A single cell was able to form BGSCs by proliferation. (Scale bars: A and D, $100 \mu \mathrm{m} ; \mathrm{B}, 50 \mu \mathrm{m}$ ).

We also determined the influence of Pax3 expression status on the proliferation of BGSCs using the CCK-8 assay. We found that the proliferation of siPax3-transfected BGSCs was significantly inhibited $(\mathrm{P}<0.05)$, but there was a significant increase in proliferation in the overexpression Pax3 plasmidtransfected BGSCs $(\mathrm{P}<0.05$; Fig. $2 \mathrm{C})$. These data suggest that Pax3 promotes the proliferation of BGSCs.

Pax3 binds the GFAP promoter. $\mathrm{Pax} 3$ is a transcription factor containing a paired domain that recognizes consensus sequences harboring a GTTCC motif and a paired-type homeodomain that binds with a consensus sequence harboring an ATTA motif (13). We used chromatin immunoprecipitation to determine whether Pax 3 can bind to the promoter region of
GFAP in the context of native chromatin. First, we analyzed the gene promoter sequences within $2.0 \mathrm{~kb}$ upstream of the transcriptional start site of genes using a pair of primers of 250-300 bp (GFAPF1, 5'-CCAGGTCCCCAGTTCATAGCA-3' and GFAP R1, 5'-TCCTTCCACATCAGCCTCCC-3'; GFAP F2, 5'-TGTCCAAATGCAGAGCATACCC-3' and GFAP R2, 5'-GGCGCAACCACGACTCACTG-3'; GFAP F3, 5'-AGG CTGAGGTGAGGGGATCA-3' and GFAP R3, 5'-AATGCT GCCAGGTCTGCTTG-3'; GFAP F4, 5'-CAAGCAGACCTG GCAGCATT-3' and GFAP R4, 5'-CTGAATAGAGCCTTG TTCTCCACC-3'; GFAP F5, 5'-CACCGGCGGTGGAGA ACAAG-3' and GFAP R5, 5'-TCTGGGGATGGATGG GTTTG-3'; GFAP F6, 5'-TCCCCAGAGGTTCTT CCCATC-3' and GFAP R6, 5'-GTGGCAGTGGAGGTCCTGATAG-3'; 
A
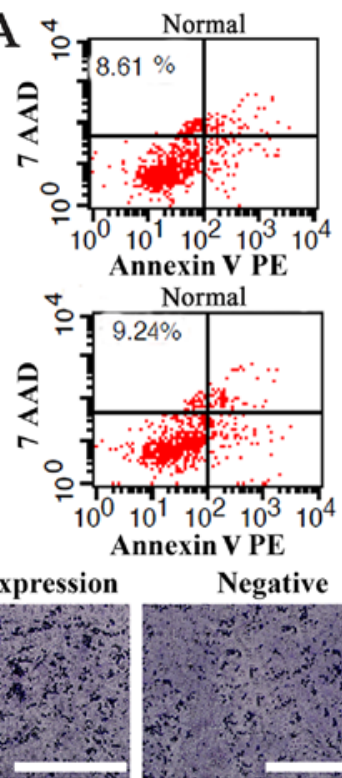

Norm
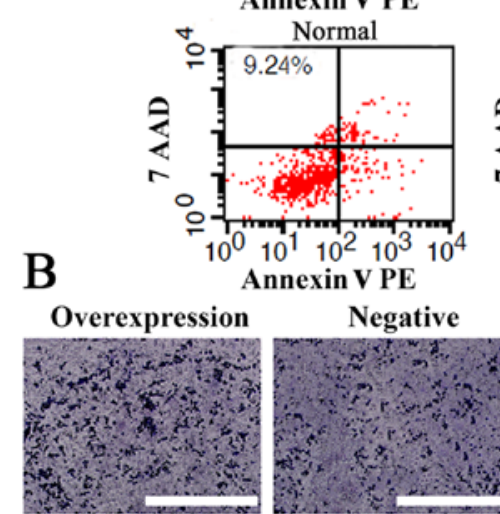

siRNA
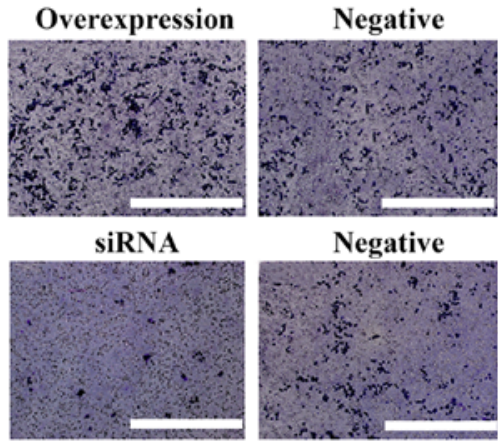

Negative

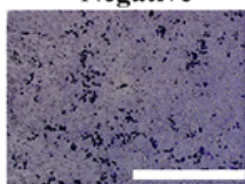

C

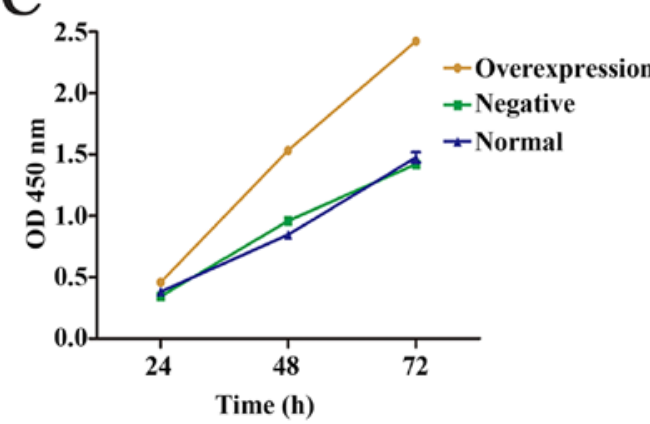

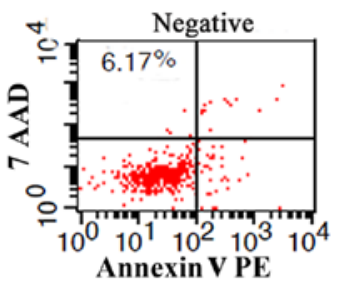

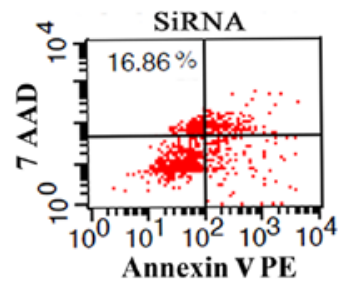

Negative
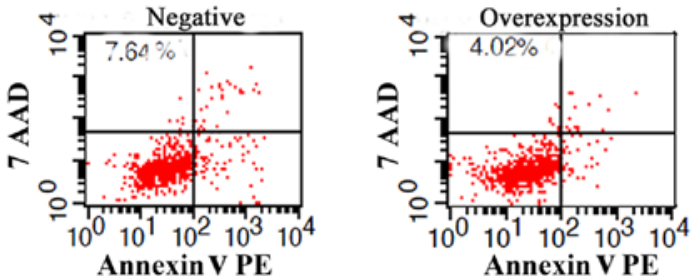

Normal
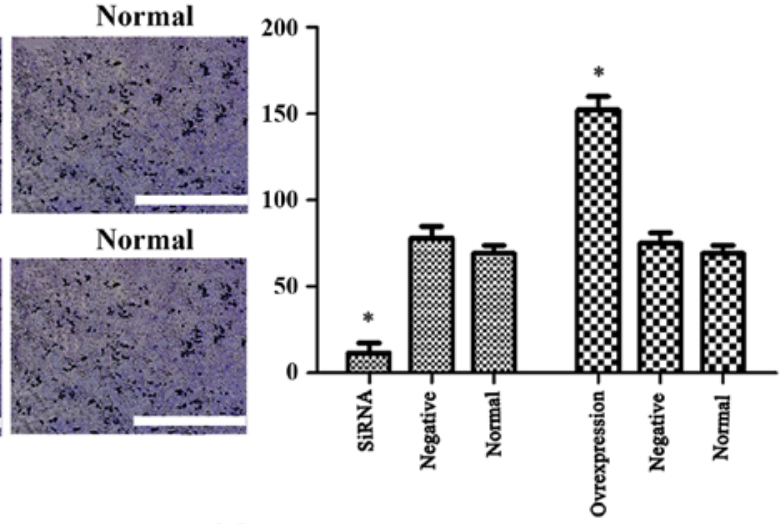

Annexin V PE

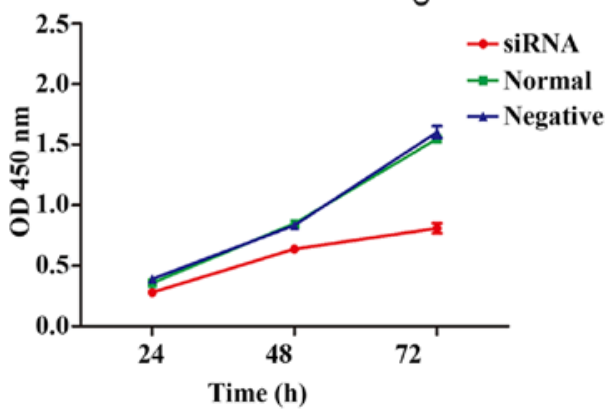

Figure 2. Effect of Pax3 overexpression on the apoptosis, cell invasion and cell proliferation of BGSCs. (A) Cells were transfected with siRNA or overexpression Pax3 plasmid and negative control or normal control cells were used. Annexin V/PI analysis was promoted to detect cell apoptosis. (B) The number of BGSCs that traversed the Matrigel basement membrane were affected by transfection with the siRNA and overexpression plasmid. (C) Effect of Pax 3 on the proliferation of BGSCs. Cells were transfected with siRNA or overexpression Pax3 plasmid, and negative and normal control cells were used. Proliferation of the cells was assessed by CCK-8 assay.

GFAP F7, 5'-CAGGACCTCCACTGCCACATAGA-3' and GFAP R7, 5'-TTTCATAACCCAGGCATTATCTCACT-3'; GFAP F8, 5'-AGTTGGAAAGCAGGTCAGAGGTCA-3' and GFAP R8, 5'-GGAAGGTGGGTCAAGAAAGGGTT-3'; GFAP F9, 5'-ACCCTTTCTTGACCCACCTTCC-3' and GFAP R9, 5'-TCTGGCTCTGCTCGCTCCTG-3'; GFAP F10, 5'-CCTCAGTGGGGTGAGGGGAGC-3' and GFAP R10, 5'-GGGGCATTCGAGCCAGGGAG-3'). We also used an anti-Pax 3 antibody specific for targeting an intron sequence of GFAP. The binding of $\mathrm{Pax} 3$ to the $\mathrm{P} 1$ and $\mathrm{P} 2$ regions was subsequently demonstrated by PCR performed with primers specific to BGSCs (Fig. 3A).

Subsequently, we referred to the study by Cao et al that described two Pax3 protein binding sites, designated P1 (5'-ATGCCAGTGAATGACTCAC-3', spanning from -949 to -979 bp on the positive strand) and P2 (5'-GGG ATTACAAGCATGAGCCACC-3', spanning from -2,172 to $-2,183 \mathrm{bp}$ on the positive strand) (18). According to these results, we constructed two recombinant normal and mutated promoters of GFAP, and performed luciferase reporter gene assays to determine mRNA transcript expression. The normal pGFAP1600 luciferase promoter construct showed low transcription activity, while inducing overexpression of Pax3 resulted in a notable increase in transcriptional activity. Furthermore, the absence of $\mathrm{P} 1$ in the promoter region resulted in a notable decrease in transcription activity, whereas the absence of the $\mathrm{P} 2$ promoter region had no obvious suppression on transcription (Fig. 3B). Together, these results indicated that: i) Pax 3 can regulate GFAP; and ii) that $\mathrm{P} 1$ is the binding region for transcription factors. Next, the EMSA revealed that Pax 3 binds to the P5 promoter region of GFAP. The double-stranded oligonucleotides corresponding to the predicted cis-element of the GFAP promoter region formed sequence-specific DNA/protein complexes with the nuclear 

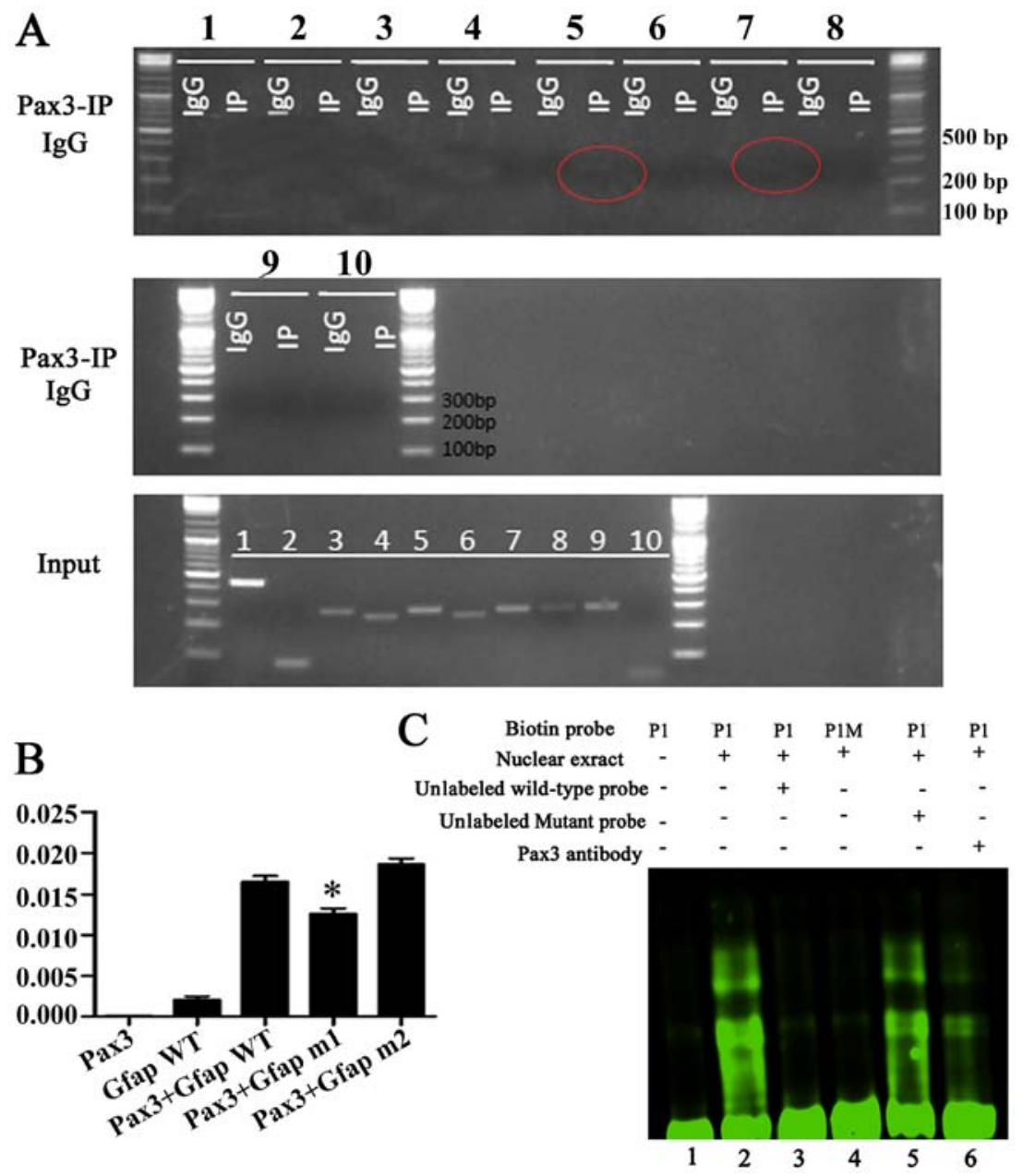

Figure 3. Pax3 binds to the P1 element in the GFAP promoter. (A) BGSCs were subjected to Chip assays with the specific anti-Pax3 antibodies and PCR primers. (B) The different human GFAP reporter (including mutated promoter) constructs were co-transfected into HEK293T cells for the luciferase assay. Relative luciferase expression levels were normalized to the empty pGL4-basic plasmid using the dual-luciferase assay system. Results are the means \pm standard deviation (SD) of the experiments performed in triplicate. (C) Nuclear extracts from the HEK293T cells expressing Pax3 were incubated with biotin-labeled probes corresponding to the P1 element, to determine whether they could form the complex. Lane 1 was the negative control with only probe. Lane 2-4, nuclear extracts from the overexpression transfected HEK293T cells. Lane 3, nuclear extracts were incubated with unlabeled wild-type probe. Lane 4, nuclear extracts were incubated with mutated P1. Lane 5, nuclear extracts were incubated with unlabeled mutant probe. Anti-Pax3 antibody was added to the reactions for supershift analysis (lane 6).

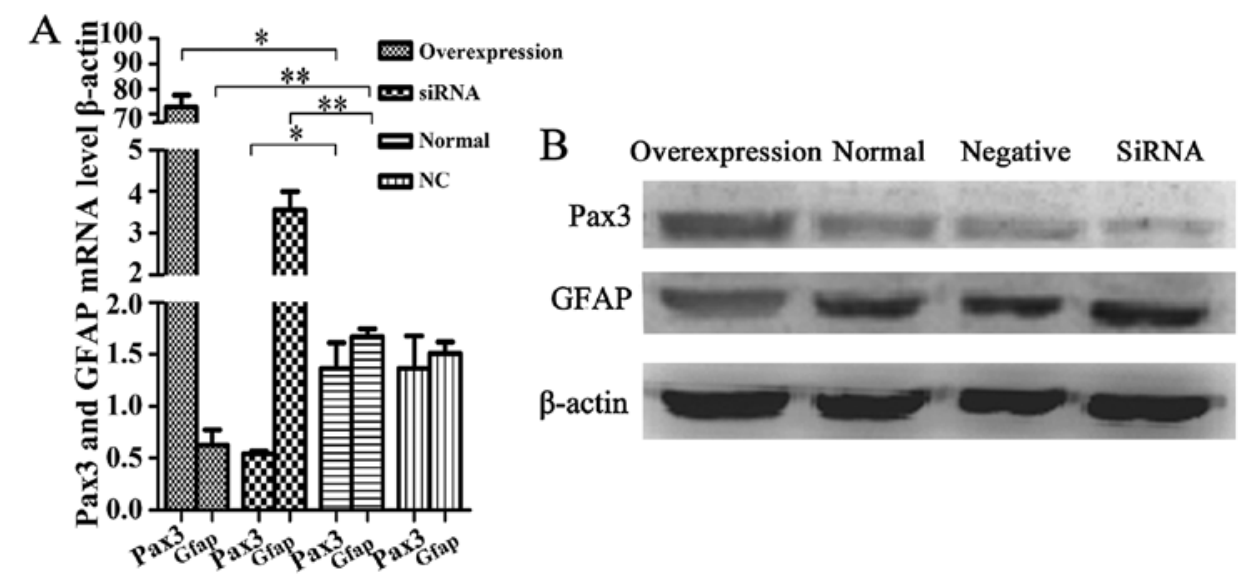

Figure 4. BGSCs were transfected with an overexpression Pax3 plasmid, siRNA, empty plasmid or no plasmid. (A) Pax3 and GFAP RNA levels were measured by quantitative RT-PCR and normalized to $\beta$-actin. (B) Pax3 and GFAP protein expression were analyzed by western blotting, and $\beta$-actin was used as a loading control $\left({ }^{*} \mathrm{P}<0.05,{ }^{* *} \mathrm{P}<0.05\right)$.

extracts of Pax3-expressing HEK293T cells, while the mutated P1 probes failed to generate such a DNA/protein complex during EMSA (Fig. 3C). Finally, we performed EMSAsupershift analysis using a fluorescently-labeled anti-Pax3 

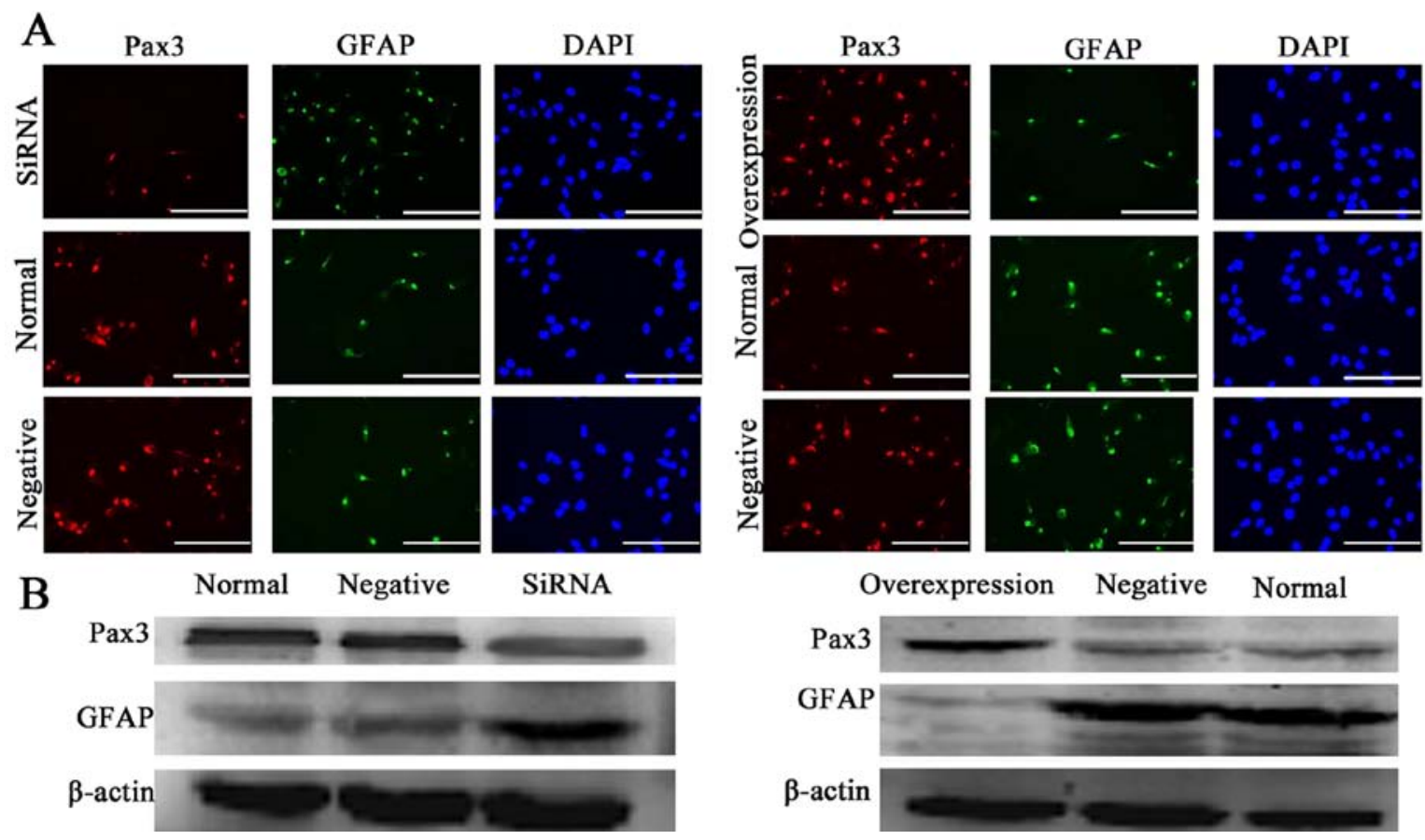

Figure 5. Pax3 modulates the differentiation of BGSCs. (A) Immunofluorescence of PAX3 and GFAP protein in the Pax3-knockdown cells and cells overexpressing Pax3 3 days post-transfection. (B) Western blotting was performed to measure Pax3 and GFAP levels in control and differentiated BGSCs. Pax3 modulated the differentiation of BGSCs. (Scale bar: A, $100 \mu \mathrm{m}$ ).

antibody to stain the nuclear extract of HEK 293T cells with overexpression of Pax3. The presence of the anti-Pax3 antibody generated the supershift band.

Pax3 inhibits GFAP expression in BGSCs and has an effect on BGSC differentiation. To ascertain that Pax3 is a negative regulator of GFAP transcription, we suppressed Pax3 expression with an siRNA that specifically targets Pax3 mRNA, while creating a Pax 3 enhanced-expression model via transfection with an overexpression-Pax 3 plasmid. Then, we performed RT-PCR and western blotting to determine the expression of GFAP at the mRNA and protein expression levels, respectively, with the Pax3 knockdown and overexpression BGSC models. We found that the expression of GFAP mRNA and protein was significantly greater after Pax 3 knockdown compared to that noted in the untransfected cells $(\mathrm{P}<0.05)$. In addition, GFAP mRNA and protein were both decreased in the Pax3-overexpression model $(\mathrm{P}<0.05)$. The results implicate that Pax3 negatively regulates the expression of GFAP at both the mRNA and protein levels (Fig. 4). To determine whether overexpression and suppression of Pax 3 modulated the differentiation of BGSCs, we transfected siRNA and an overexpression-Pax3 plasmid into the BGSCs. We performed immunofluorescence with anti-GFAP and showed that the proportion of GFAP-positive cells was 20, 24 and $67 \%$ in the negative controls, untransfected cells and BGSCs transfected with siPax3, respectively. We also observed that, in association with Pax3 silencing via siRNA, the expression of GFAP significantly increased compared to the untransfected cells $(\mathrm{P}<0.05$; Fig. 5A). Furthermore, western blotting also showed that the expression of GFAP protein in the BGSCs was significantly increased after the knockdown of Pax3 compared to the untransfected cells $(\mathrm{P}<0.05$; Fig. 5B). These results demonstrated that Pax3 negatively regulates the expression of GFAP and affects the differentiation in BGSCs.

\section{Discussion}

Brain glioma stem cells (BGSCs) were initially isolated from leukemic cells (19). Following Reya et al recognition of the similarities in the biological hallmarks of stem and tumor cells, it was proposed that cancers may evolve from a subset of precursor stem cells (20). Since then, stem cells have been isolated from the tumor microenvironment of numerous types of cancers, including those of the nasopharynx, breast and prostate cancer (21). Although Ignatova et al previously reported on the isolation of BGSCs from cortical glial tumors (22), it is apparent that we currently know very little in regards to the contribution of BGSCs to tumorigenesis. Accordingly, the present study endeavored to investigate the putative roles of BGSCs, with particular prominence on their contribution to the hallmarks of cancers.

$\mathrm{CD}_{133^{+}}$cells exist in various tissues, including umbilical cord blood, fetal brain, fetal liver and placental trophoblasts. Moreover, immunohistochemical staining has shown that CD133 is distributed throughout stem cells; on the membrane and within the cytoplasm. Recent studies have shown that CD133 is expressed in neural stem cells (NSCs) $(23,24)$. Furthermore, nestin, a protein marker for NSCs, is also expressed in follicle stem cells. In the present study, by immunohistochemical staining, we identified CD133 and nestin as putative markers of BGSCs.

Prior research has implicated that Pax 3 binds to the promoter region of GFAP, thereby negatively regulating GFAP expression and subsequently arresting the differentiation of 
astrocytes from NSCs (13). Furthermore, the overexpression of Pax3 has been observed within glioma tissues, in which it was reported to regulate GFAP expression in glioma cells (15). However, no studies have focused on the transcriptional relationship between Pax3 and GFAP in BGSCs. Herein, we found that Pax3 was expressed at a higher level in BGSCs than in U87MG malignant glioma cell and 1800 normal astrocyte lines. Then, we identified a binding element within the promoter region of GFAP to which Pax 3 could associate, and subsequently demonstrated that $\mathrm{Pax} 3$ inhibits the transcription of GFAP in BGSCs.

To describe the influence of $\mathrm{Pax} 3$ on the behavior of BGSCs, Pax3 was silenced via the transfection of small interfering RNA (siRNA) into BGSCs. The subsequent cell proliferation, invasive and apoptosis assays showed that this siRNA-induced downregulation of Pax3 inhibited proliferation, induced apoptosis and decreased the invasiveness of transfected BGSCs. In contrast, upregulation of Pax 3 via transfection of BGSCs with an overexpression vector induced increased cell proliferation and invasiveness, while suppressing apoptosis. Together, these results suggests that Pax3 plays a vital role in the growth and evolution of BGSCs.

To further determine the effects of Pax 3 on BGSC differentiation and GFAP expression, siPax3-transfected BGSCs were cultivated in culture medium containing serum, to which the ability of BGSCs to differentiate was decreased. To the contrary, differentiation was increased in the overpression Pax3 plasmid-transfected BGSCs. Our results indicate that Pax3 acts as a transcriptional repressor during seruminduced differentiation of BGSCs. Thereby, it is reasonable to speculate that Pax3 suppresses the expression of GFAP during oncogenesis.

In conclusion, we firstly demonstrated that Pax 3 binds to the promoter region of $G F A P$, and consequently negatively regulates GFAP expression in BGSCs. Pax3 can be considered a regulator of BGSC differentiation and may determine the degree of the malignancy of gliomas. Pax3 also plays a crucial role in the regulation of the growth and invasion of BGSCs. The results of the present study imply that Pax 3 is a putative target for novel therapies endeavoring to treat gliomas.

\section{Acknowledgements}

Brain glioma stem cells were kindly gifted by Soochow University, China. The present study was supported by the Natural Science Foundation of Jiangsu Province (BK20130386), the National Natural Science Foundation of China (81402447), the Chinese Projects for Postdoctoral Science Funds (no. 2015M571792), the Jiangsu Planned Projects for Postdoctoral Research Funds (no. 1402200C), the Six Major Human Resources Project of Jiangsu Province (2014-WSW-028), and the Technology Project of Nantong (HS2014053).

\section{References}

1. Fang JS, Deng YW, Li MC, Chen FH, Wang YJ, Lu M, Fang F Wu J, Yang ZY, Zhou XY, et al: Isolation and identification of brain tumor stem cells from human brain neuroepithelial tumors. Zhonghua Yi Xue Za Zhi 87: 298-303, 2007 (In Chinese).
2. Stupp R, Mason WP, van den Bent MJ, Weller M, Fisher B, Taphoorn MJ, Belanger K, Brandes AA, Marosi C, Bogdahn U, et al; European Organisation for Research and Treatment of Cancer Brain Tumor and Radiotherapy Groups; National Cancer Institute of Canada Clinical Trials Group: Radiotherapy plus concomitant and adjuvant temozolomide for glioblastoma. $\mathrm{N}$ Engl J Med 352: 987-996, 2005.

3. Komotar RJ, Otten ML, Moise G and Connolly ES Jr: Radiotherapy plus concomitant and adjuvant temozolomide for glioblastoma-a critical review. Clin Med Oncol 2: 421-422, 2008.

4. Enting RH, van der Graaf WT, Kros JM, Heesters M, Metzemaekers J and den Dunnen W: Radiotherapy plus concomitant and adjuvant temozolomide for leptomeningeal pilomyxoid astrocytoma: A case study. J Neurooncol 80: 107-108, 2006.

5. Frosina G: Stem cell-mediated delivery of therapies in the treatment of glioma. Mini Rev Med Chem 11: 591-598, 2011.

6. Dietrich J, Diamond EL and Kesari S: Glioma stem cell signaling: Therapeutic opportunities and challenges. Expert Rev Anticancer Ther 10: 709-722, 2010.

7. Bonnet D and Dick JE: Human acute myeloid leukemia is organized as a hierarchy that originates from a primitive hematopoietic cell. Nat Med 3: 730-737, 1997.

8. Singh SK, Hawkins C, Clarke ID, Squire JA, Bayani J, Hide T, Henkelman RM, Cusimano MD and Dirks PB: Identification of human brain tumour initiating cells. Nature 432: 396-401, 2004.

9. Galli R, Binda E, Orfanelli U, Cipelletti B, Gritti A, De Vitis S, Fiocco R, Foroni C, Dimeco F and Vescovi A: Isolation and characterization of tumorigenic, stem-like neural precursors from human glioblastoma. Cancer Res 64: 7011-7021, 2004.

10. Plummer RS, Shea CR, Nelson M, Powell SK, Freeman DM, Dan CP and Lang D: PAX3 expression in primary melanomas and nevi. Mod Pathol 21: 525-530, 2008.

11. Ryu B, Kim DS, Deluca AM and Alani RM: Comprehensive expression profiling of tumor cell lines identifies molecular signatures of melanoma progression. PLoS One 2: e594, 2007.

12. Liu F, Cao J, Lv J, Dong L, Pier E, Xu GX, Wang RA, Xu Z, Goding $\mathrm{C}$ and Cui R: TBX2 expression is regulated by PAX3 in the melanocyte lineage. Pigment Cell Melanoma Res 26: 67-77, 2013.

13. Liu Y, Zhu H, Liu M, Du J, Qian Y, Wang Y, Ding F and Gu X: Downregulation of Pax3 expression correlates with acquired GFAP expression during NSC differentiation towards astrocytes. FEBS Lett 585: 1014-1020, 2011.

14. Gomes FC, Paulin D and Moura Neto V: Glial fibrillary acidic protein (GFAP): Modulation by growth factors and its implication in astrocyte differentiation. Braz J Med Biol Res 32: 619-631, 1999.

15. Chen J, Xia L, Wu X, Xu L, Nie D, Shi J, Xu X, Ni L, Ju S, Wu X, et al: Clinical significance and prognostic value of PAX3 expression in human glioma. J Mol Neurosci 47: 52-58, 2012.

16. Xia L, Huang Q, Nie D, Shi J, Gong M, Wu B, Gong P, Zhao L, Zuo H, Ju S, et al: PAX3 is overexpressed in human glioblastomas and critically regulates the tumorigenicity of glioma cells. Brain Res 1521: 68-78, 2013.

17. Cebolla B and Vallejo M: Nuclear factor-I regulates glial fibrillary acidic protein gene expression in astrocytes differentiated from cortical precursor cells. J Neurochem 97: 1057-1070, 2006.

18. Cao L, Yu Y, Bilke S, Walker RL, Mayeenuddin LH, Azorsa DO, Yang F, Pineda M, Helman LJ and Meltzer PS: Genome-wide identification of PAX3-FKHR binding sites in rhabdomyosarcoma reveals candidate target genes important for development and cancer. Cancer Res 70: 6497-6508, 2010.

19. Blair A, Hogge DE, Ailles LE, Lansdorp PM and Sutherland HJ: Lack of expression of Thy-1 (CD90) on acute myeloid leukemia cells with long-term proliferative ability in vitro and in vivo. Blood 89: 3104-3112, 1997.

20. Reya T, Morrison SJ, Clarke MF and Weissman IL: Stem cells, cancer, and cancer stem cells. Nature 414: 105-111, 2001.

21. Wang J, Guo LP, Chen LZ, Zeng YX and Lu SH: Identification of cancer stem cell-like side population cells in human nasopharyngeal carcinoma cell line. Cancer Res 67: 3716-3724, 2007.

22. Ignatova TN, Kukekov VG, Laywell ED, Suslov ON, Vrionis FD and Steindler DA: Human cortical glial tumors contain neural stem-like cells expressing astroglial and neuronal markers in vitro. Glia 39: 193-206, 2002.

23. Rafii S: Circulating endothelial precursors: Mystery, reality, and promise. J Clin Invest 105: 17-19, 2000.

24. Uchida N, Buck DW, He D, Reitsma MJ, Masek M, Phan TV, Tsukamoto AS, Gage FH and Weissman IL: Direct isolation of human central nervous system stem cells. Proc Natl Acad Sci USA 97: 14720-14725, 2000. 